\title{
La Música como promotora de habilidades fonológicas: un estudio exploratorio con niños preescolares uruguayos
}

\section{Music as a promoter of phonological skills: an exploratory study with Uruguayan preschool children}

\section{A música como promotora de habilidades fonológicas: um estudo exploratório com pré-escolares uruguaios}

\author{
Johanna Rivera Ibaceta ${ }^{1}$, ORCID 0000-0001-9767-819X \\ Karen Moreira Tricot ${ }^{2}$, ORCID 0000-0002-6496-674X \\ ${ }^{12}$ Facultad de Psicología, Universidad de la República. Uruguay
}

Resumen: Este estudio presenta los resultados de un Programa de estimulación de la Conciencia Fonológica (CF), a través de la música en un grupo de 21 niños uruguayos de 5 años de edad. Se utilizó un diseño cuasiexperimental de comparación de grupos (pre-postestseguimiento). Los niños que participaron del Programa de estimulación obtuvieron un puntaje mayor que los niños del grupo control en tareas de $\mathrm{CF}$, al finalizar el periodo preescolar, y, además, se desempeñaron mejor en una tarea de lectura de palabras que se les administró al finalizar el primer año de escuela. Los resultados se discuten tomando en cuenta lo reportado por la literatura internacional que sostiene que participar de programas de entrenamiento musical favorece el desarrollo de las habilidades de $\mathrm{CF}$, y en función de las características del programa de estimulación que estuvo centrado particularmente en el desarrollo de habilidades vinculadas con el ritmo, la precisión y la duración.

Palabras clave: conciencia fonológica; habilidades musicales; adquisición de la lectura; programa de estimulación musical; ritmo

Abstract: In this study we describe the effects of a Music Intervention Program on the Phonological Awareness, PA, of a group of 21 5-year-old Uruguayan children. A quasiexperimental design of pre-post test group comparison was used. At the end of the preschooling period, children who participated in the Music Intervention Program performed better in Phonological Awareness tasks than those of the control group. Finally, in order to analyze the reading performance of the subjects, a word-reading test was administered at the end of the first year of school. The analysis showed statistically significant differences between the groups in reading efficiency. The discussion of the results is based on the characteristics of the stimulation program, which was focused on the development of skills related to rhythm, accuracy and duration. The discussion also takes into account reports from current international literature, which asserts that taking part in a Music Intervention Program stimulates the development of PA Skills.

Keywords: Phonological awareness; musical skills; reading acquisition; preschoolers; music intervention program; rhythm 
Resumo: Este estudo apresenta os resultados de um Programa de Estimulação da Consciência Fonológica (CF), através da música em um grupo de 21 crianças uruguaias de 5 anos. Foi utilizado um desenho de comparação de grupos, quase experimental (pré-pós-follow-up). As crianças que participaram do programa de estimulação obtiveram uma pontuação maior do que as crianças do grupo controle nas tarefas de CF no final do período pré-escolar e, além disso, tiveram melhor desempenho em uma tarefa de leitura de palavras que foi administrada no final do primeiro período do ensino fundamental. Os resultados são discutidos levando em consideração o que foi relatado pela literatura internacional que sustenta que a participação em programas de treinamento em música favorece o desenvolvimento das habilidades da $\mathrm{CF}$, e contemplando as características do programa de estimulação que foi particularmente focado no desenvolvimento de habilidades ligado ao ritmo, precisão e duração.

Palavras-chave: consciência fonológica; habilidades musicais; aquisição de leitura; programa de estimulação musical; ritmo

Recibido: 04/04/2020

Aceptado: $12 / 08 / 2020$

Cómo citar:

Rivera Ibaceta, J., \& Moreira Tricot, K. (2020). La Música como promotora de habilidades fonológicas: un estudio exploratorio con niños preescolares uruguayos. Ciencias Psicológicas, 14(2), e-2270. doi: https://doi.org/10.22235/cp.v14i2.2270

Correspondencia: Johanna Rivera Ibaceta, Instituto de Fundamentos y Métodos en Psicología, Facultad de Psicología, Universidad de la República, UdelaR, Uruguay. E-mail: jrivera@psico.edu.uy. Karen Moreira Tricot, Instituto de Fundamentos y Métodos en Psicología, Facultad de Psicología, Universidad de la República, UdelaR, Uruguay. E-mail: moreirak@psico.edu.uy

La Conciencia Fonológica, CF, es una habilidad metalingüística compleja que permite el análisis de cualquier unidad fonológica del lenguaje oral, y se considera uno de los precursores clave para aprender a leer en lenguas que cuentan con un sistema de representación alfabético (Alegría, 2006; Caravolas et al., 2012; Castles, Rastle, \& Nation, 2018; Defior, 2014; Dehaene, 2017; Melby-Lervåg, Lyster, \& Hulme, 2012).

El desarrollo de la CF progresa desde la sensibilidad para reflexionar sobre las unidades más grandes de la cadena sonora hablada (palabras), hasta alcanzar el nivel de mayor complejidad que implica identificar las unidades más pequeñas (fonemas) que conforman el lenguaje oral (Defior \& Serrano, 2014; Melby-Lervåg et al., 2012; Nohales \& Giménez, 2014).

La posibilidad de identificar segmentos cada vez más pequeños en el habla depende tanto del desarrollo cognitivo del sujeto, como del medio social en que vive. Ciertos niveles de CF tienden a adquirirse precozmente, mientras otros emergen durante el proceso de aprendizaje de la lectura (Defior, 2014; Defior \& Serrano, 2014; Defior \& Serrano, 2011; de la Calle Cabrera, Villagrán, \& Guzmán, 2016; Domínguez, Nasini, \& Teberosky, 2013; Melby-Lervåg et al., 2012; Nohales \& Giménez, 2014; Villagrán, Consejero, Guzmán, Jiménez, \& Cuevas, 2011). En ese sentido, a partir de los 4 años, los niños, cuyo contexto alfabetizador es favorable, pueden reconocer que dos palabras que tienen significados diferentes comparten sus sonidos al final. 
Identificar que dos palabras terminan igual es uno de los primeros conocimientos que el niño tiene sobre la estructura sublexical de la palabra. Este tipo de conocimiento requiere una manipulación de segmentos menos consciente y deliberada que los otros niveles, lo que hace que su demanda cognitiva sea menor si se compara con otras habilidades fonológicas, y por ello, en la mayoría de los casos, no depende de la enseñanza explícita.

Posteriormente, los niños son capaces de someter a análisis las unidades articulatorias mínimas del lenguaje pudiendo realizar tareas fonológicas que demandan identificar y manipular las sílabas de una palabra. Más tarde, pueden analizar la sílaba y descomponerla en sus unidades de ataque y rima intrasilábica, y pueden realizar tareas que requieren aislar o identificar sonidos iniciales o finales en una palabra. Hacia los 6 años, momento en que entran en contacto directo con el aprendizaje formal de la lectura y de las Reglas de Conversión Grafema Fonema, RCGF, pueden reflexionar y operar plenamente con todas y cada una de las unidades mínimas y abstractas del lenguaje oral: los fonemas (Cuadro, 2010; Defior \& Serrano, 2014; Castles et al., 2018; Torres, 2014). Cada una de estas habilidades fonológicas comporta distintos niveles de dificultad según las demandas cognitivas, lingüísticas, analíticas y de memoria que requieran las tareas.

Un nivel de complejidad, proviene de la posición de las unidades a identificar en la palabra. Para los niños que aún no han aprendido a leer reconocer unidades que se encuentran en posición inicial resulta más sencillo que operar con unidades que se encuentran en posición final (ej. aislar sonido en posición inicial versus aislar sonido en posición final). Por el contrario, si se trata de una tarea que requiere manipular unidades el grado de complejidad es mayor si la unidad a manipular se encuentra en posición inicial que en posición final (ej. omitir sonido en posición inicial versus omitir sonido en posición final). El desempeño de los niños en tareas fonológicas de omisión y de adición de unidades fonológicas mejora considerablemente cuando ingresan a primer año de educación primaria, lo que sugiere la necesidad del aprendizaje lector para que éstas se realicen correctamente, aun cuando pueda observarse un porcentaje de niños que pueden resolver estas tareas a los 5 años. El nivel de complejidad mayor, para cualquier tipo de tarea, se observa en la identificación y manipulación de unidades que se encuentran en posición medial (Bravo, Villalón \& Orellana, 2011; de la Calle Cabrera et al., 2016; Nohales \& Giménez, 2014).

La literatura muestra que es posible estimular la CF a través de la instrucción directa en tareas de análisis fonológico, mediante juegos con el lenguaje oral, lectura de cuentos, rimas y canciones infantiles, y que este entrenamiento produce mejoras en el aprendizaje de la lectura tanto en niños prelectores con desarrollo típico como en niños que experimentan dificultades durante el proceso lector (Cuadro \& Trías, 2008; Gordon, Fehd, \& McCandliss, 2015; Jiménez \& Ortiz, 2008; Kjeldsen, Karna, Niemi, Olofsson, \& Witting, 2014).

Adicionalmente, existe evidencia sobre el papel que juegan las habilidades musicales en el desarrollo de habilidades lingüísticas, y en particular de la CF en distintas lenguas (Anvari, Trainor, Woodside, \& Levy, 2002; Bolduc \& Montésinos-Gelet, 2005; Gordon et al., 2015; Hansen, Bernstorf, \& Stuber, 2014; Kraus \& Chandrasekaran, 2010; Lamb \& Gregory, 1993; Lucas \& Gromko, 2007; Peynircioğlu, Durgunoglu, \& ÚneyKüsefog`lu, 2002; Tierney \& Kraus, 2013). Varios estudios correlacionales han mostrado que existe una asociación entre las habilidades musicales y las habilidades de CF, debido a que el análisis auditivo que se realiza para la percepción de los elementos rítmicos, armónicos y melódicos de una estructura musical es similar al procesamiento del lenguaje oral, al segmentar el flujo del habla y manipular unidades fonológicas (Gordon et al., 2015; Goswami, 2012a; Kraus \& Chandrasekaran, 2010; Patel, 2010; Tierney \& Kraus, 2013).

Uno de los estudios pioneros en este campo fue el de Lamb y Gregory (1993), quienes evaluaron a un grupo de 16 niños de 4 y 5 años en habilidades lectoras, nivel de conciencia fonémica y en habilidades musicales de discriminación melódica y tímbrica. Sus resultados mostraron una asociación entre la conciencia fonémica y las habilidades lectoras, y, a la vez, 
indicaron que la discriminación melódica tuvo una correlación significativa con la conciencia fonémica. Años más tarde, Anvari et al. (2002), administraron una batería de tareas musicales y fonológicas a un grupo de 100 niños canadienses de habla inglesa de 4 y 5 años. Los análisis de regresión mostraron que las habilidades de percepción musical contribuyeron, con una varianza única, a la predicción de la habilidad lectora, aun cuando la varianza debida a la CF y otras habilidades cognitivas (matemáticas, memoria y vocabulario) fueron controladas. En los niños de 4 años las habilidades rítmicas y melódicas fueron predictoras de la $\mathrm{CF}$, mientras que en los niños de 5 años sólo las habilidades melódicas se asociaron positivamente con ésta.

Peynircioğlu et al. (2002), reportaron una asociación positiva entre el buen desempeño en tareas de percepción melódica y rítmica y los puntajes obtenidos en tareas de CF y de reconocimiento de pseudopalabras en niños turcos norteamericanos, independientemente de la lengua materna de los sujetos. Por su parte Bolduc y Montésinos-Gelet (2005), analizaron el desempeño de un grupo de preescolares canadienses de habla francesa en tareas de percepción musical de elementos melódicos y rítmicos y en tareas fonológicas, y encontraron una correlación positiva entre el desempeño en tareas de percepción melódica y de identificación de sílabas y fonemas iniciales, así como en la identificación de rimas, aunque la correlación entre tareas rítmicas y CF fue menor. Lucas y Gromko (2007), condujeron un estudio con un grupo de niños norteamericanos de 6 años y encontraron una correlación significativa entre el desempeño en tareas de discriminación melódica y rítmica, y en la tarea de segmentación de fonemas en el primer año de educación primaria.

Se suman a esta evidencia los estudios que han mostrado que participar activamente de programas de música tiene efectos beneficiosos en el desarrollo de habilidades lingüísticas de niños prelectores, ya que el entrenamiento musical es una actividad exigente que, además de la percepción auditiva, implica la manipulación y el trabajo con estructuras temporales y sonoras no lingüísticas que se suceden en el tiempo. Un tipo de operación similar a la que demanda la resolución de una tarea fonológica que, además de analizar los sonidos del habla, requiere de un manejo eficiente de las propiedades temporales inherentes a la estructura de las unidades lingüísticas. Por esta razón sujetos que presentan un déficit en el procesamiento temporal suelen tener dificultades para aprender a leer y para desempeñarse con éxito en ejercicios musicales de reproducción de patrones rítmicos (Bolduc, 2009; Degé \& Schwarzer, 2011; Dellatolas et al., 2009; Flaugnacco et al., 2014; François, Chobert, Besson, \& Schön, 2012; Goswami, 2012b; Gromko, 2005; Moritz, Yampolsky, Papadelis, Thomson, \& Wolf, 2013; Peretz, 2018; Slater at al., 2014; Tierney \& Kraus, 2013).

En España, Herrera, Defior, y Lorenzo (2007), llevaron adelante un estudio con niños preescolares de habla hispana y niños que hablaban tamazight, una lengua de transmisión oral que se habla en la zona Rif de Marruecos. Si bien el objetivo fue evaluar la eficacia de dos programas de entrenamiento en $\mathrm{CF}$, en uno de los grupos de tratamiento se utilizaron las mismas palabras para trabajar los distintos niveles de CF, pero a través de canciones infantiles. Luego de dieciséis sesiones los dos grupos que fueron entrenados tuvieron un desempeño superior al de los controles en $\mathrm{CF}$, independientemente de su lengua. Además, los niños que participaron del programa que utilizaba canciones infantiles incrementaron su desempeño en las tareas de identificación de la rima léxica.

Gromko (2005) comparó el desempeño en algunas tareas de CF entre dos grupos de preescolares antes y después de que uno de ellos participara de un programa de música durante 16 semanas. Los niños entrenados obtuvieron un desempeño superior al de los del grupo control en reconocimiento de letras y en tareas de segmentación.

Bolduc (2009) comparó el desempeño en habilidades fonológicas de un grupo de niños franco-canadienses expuestos a dos programas de música. El grupo experimental participó de un entrenamiento específico en habilidades melódicas y rítmicas, mientras el grupo control asistió a las clases curriculares de música ofrecidas por su escuela. Ambos programas de música fueron 
efectivos para estimular habilidades de percepción musical, pero sólo el entrenamiento específico tuvo un efecto en habilidades de CF.

Degé y Schwarzer (2011), compararon los efectos de un programa de entrenamiento de la $\mathrm{CF}$, y un programa de entrenamiento musical en habilidades fonológicas en un grupo de niños preescolares. Un grupo participó de un programa de entrenamiento directo en $\mathrm{CF}$, otro grupo participó de un programa de música, y un grupo control realizó actividades deportivas. Luego de 20 semanas de entrenamiento los dos grupos que fueron entrenados en CF mejoraron sus desempeños en tareas fonológicas, a diferencia de los sujetos del grupo control que mantuvieron su rendimiento inicial.

Moritz y colegas (2013), llevaron a cabo un estudio longitudinal en el que analizaron la relación entre las habilidades rítmicas y la CF en un grupo de 30 niños preescolares. Se comparó el desempeño entre dos grupos expuestos a programas de intervención musical que diferían en sus actividades musicales y en su carga horaria. El grupo experimental participó diariamente de un entrenamiento basado en el método de enseñanza musical Kodály, durante todo el año escolar. Mientras el grupo control recibió una instrucción centrada en interpretar canciones infantiles, y trabajó sobre nociones musicales básicas tales como, lento rápido, grave y agudo, entre otras. Los niños que recibieron el entrenamiento diariamente tuvieron un desempeño superior en tareas de $\mathrm{CF}$ al final del año escolar. Dos años más tarde, 12 niños de la muestra inicial (8 pertenecientes al grupo experimental y 4 del grupo control en el nivel inicial), fueron nuevamente evaluados en tareas fonológicas. El análisis de los datos mostró que las habilidades rítmicas adquiridas en el preescolar correlacionaron con las habilidades fonológicas en el segundo año de escuela.

Slater y colegas (2014), realizaron un estudio longitudinal con 42 niños bilingües (español - inglés) de 6 y 9 años, provenientes de un nivel socioeconómico bajo, y analizaron el impacto del entrenamiento musical en las habilidades lectoras en niños que ven afectado su rendimiento lector por las características de su entorno sociocultural. Se administró una batería de test para medir habilidades lectoras y nivel de inteligencia, además se procuró que todos los sujetos fueran semejantes en edad, género, edad de adquisición del inglés, competencia lectora en inglés y nivel educacional de los padres. Del conjunto de niños evaluados, 23 sujetos conformaron el grupo experimental e iniciaron el entrenamiento inmediatamente después de las evaluaciones, mientras los 19 niños restantes actuaron como grupo control pasivo. Luego de un año de entrenamiento musical no se observaron mejoras en el rendimiento lector, ni en CF, pero los niños entrenados mantuvieron el nivel de lectura normal acorde a su edad, mientras que los sujetos del grupo control vieron disminuido su desempeño en lectura durante ese mismo periodo. Los autores discutieron estos resultados argumentando que el deterioro en las habilidades lectoras observadas en los niños sin entrenamiento es consistente con la trayectoria negativa de rendimiento que se observa en la población de niños en situación de vulnerabilidad, y que en ese sentido la participación en el programa de música contrarrestó el impacto negativo. Al mismo tiempo, discutieron la no existencia de diferencias significativas entre los grupos en $\mathrm{CF}$, en función de que la experiencia bilingüe temprana puede conferir una ventaja para la CF.

La mayor parte de estas investigaciones se han llevado a cabo con niños de habla inglesa, o de lenguas diferentes al español, existiendo un número considerablemente menor de estudios sobre la estimulación de la CF a través de la música en niños de habla hispana. Al mismo tiempo, se observa que el desarrollo de habilidades rítmicas, en la estructuración de actividades musicales ha estado menos presente, aun cuando se ha sugerido que la percepción del ritmo del habla facilita el reconocimiento de la palabra hablada y el desarrollo del vocabulario en los niños, promoviendo el desarrollo de la CF (Holliman, Wood, \& Sheehy, 2010; Wood, Wade-Woolley, \& Holliman, 2009). En efecto, niños que presentan bajos niveles de CF, o que presentan dificultades en el proceso de adquisición de la lectura también presentan dificultades en las habilidades rítmicas y de sincronización en tareas musicales (Goswami, 2012b; Goswami, Wang, 
Cruz, Fosker, Mead, \& Huss, 2010; Flaugnacco et al., 2014; Lundetræ \& Thomson, 2018; Moritz et al., 2013; Tierney \& Kraus, 2013; Tierney, White-Schwoch, MacLean, \& Kraus, 2017; Thomson, Huss \& Goswami, 2006).

A la fecha, no encontramos estudios en español que evalúen los resultados de un programa de estimulación de la $\mathrm{CF}$, a través de actividades musicales enfocadas en el ritmo, en niños preescolares con desarrollo típico. Resulta entonces de la mayor relevancia generar evidencia empírica específica para esta población. El objetivo principal de este estudio fue analizar los efectos de un Programa de estimulación de la $\mathrm{CF}$, a través de una intervención musical centrada en el desarrollo de habilidades rítmicas, en un grupo de niños uruguayos de 5 años, a los efectos de extender los hallazgos de otras lenguas al español de Uruguay.

\section{Materiales y Método}

\section{Participantes}

Al inicio del estudio se contó con la participación de 26 niños (10 niñas y 16 niños). Los datos de 5 niños (2 niños del GE y 3 niños del GC) fueron excluidos de los análisis estadísticos, por razones diversas: dos niños no pudieron sostener las pruebas de evaluación en la fase de pretest. Particularmente no completaron la primera parte de la Prueba de Segmentación Lingüística (ver descripción en instrumentos) que, por su extensión y complejidad, debe aplicarse en dos sesiones; una participante asistió a menos del 50\% de las actividades de estimulación; un niño, debido a problemas familiares, vio afectado su desempeño y participación tanto en el programa de intervención como en su clase (situación que fue informada por la maestra); y un niño, a fin de año fue diagnosticado con síndrome de Asperger leve. Por lo tanto, la muestra final estuvo formada por 21 niños (8 niñas y 13 niños), con un rango de edad de 61 a 70 meses $(M=65.95, D E=2.97)$, inscriptos en el último año de Educación inicial de una escuela pública en la ciudad de Montevideo en Uruguay, categorizada por el sistema educativo uruguayo como de quintil 5 (proxy de NSE medio). La lengua materna de los niños era el español, no presentaban diagnóstico de ningún trastorno ligado al desarrollo cognitivo o linguiístico, y tenían una asistencia regular al centro educativo. Todos provenían de la misma aula, y estaban cursando el nivel educativo esperado para su edad. Los participantes no tenían clases de música dentro del programa escolar, ni asistían a talleres extracurriculares en esta disciplina.

Esta investigación contó con la aprobación del Comité de ética de la Facultad de Psicología de la Universidad de la República, y se ajustó al Decreto CM/515 del Poder Ejecutivo sobre Investigación con Seres Humanos, amparándose en los procedimientos legales para la protección de la identidad de los participantes. Al mismo tiempo, se solicitó consentimiento informado a los padres y asentimiento a los niños para participar de este estudio.

\section{Procedimiento}

En el pretest (Tiempo 1) se administraron en sesiones individuales una batería de pruebas que se describe a continuación:

1. Test de Matrices Progresivas Raven (MPR), escala coloreada, (Raven \& Court, 1993). Se trata de un instrumento de screening que mide el nivel de inteligencia fluida en niños a través de la resolución de tareas de razonamiento no verbal con base en estímulos figurativos. Consta de tres series de matrices de 12 items cada una ( $\mathrm{A}, \mathrm{AB}$ y $\mathrm{B}$ ). Cada serie integra una escala de matrices en orden de complejidad creciente.

2. Test de vocabulario en imágenes, Peabody (PPVT), (Dunn \& Dunn, 2006). Corresponde a una prueba clásica de vocabulario receptivo, que evalúa la comprensión verbal. La prueba consta de 192 láminas que contienen cuatro dibujos cada una, y en las que el sujeto debe indicar cuál de las 
imágenes se corresponde con el significado de la palabra que se le presenta oralmente.

3. Prueba de Segmentación Lingüística (PSL), (Jiménez \& Ortiz, 2008). Se trata de una prueba estandarizada para evaluar el Nivel de Conciencia Fonológica, NCF, durante el periodo de educación inicial. Está compuesto por una serie de tareas fonológicas, y aunque incluye tareas de segmentación léxica, predominan tareas de evaluación de la conciencia silábica, las que se describen a continuación:

\subsection{Segmentación léxica y silábica}

3.1.1 Segmentación léxica: en esta tarea se le presentan al niño oralmente unas frases y éste debe indicar el número de palabras que contiene cada enunciado.

3.1.2 Segmentar palabras en sílabas: en esta tarea se presentan oralmente palabras aisladas, y el niño debe contar el número de sílabas que componen la palabra.

\subsection{Aislar sílabas y sonido consonántico}

En esta tarea se le presenta al niño una serie de dibujos, y éste debe señalar el dibujo que comienza o termina con el fonema o sílaba que se le indica.

\subsection{Omisión de sílabas y sonido consonántico}

3.3.1 Omisión de fonema inicial: esta tarea se le presenta al niño una serie de dibujos y éste debe pronunciar el nombre de cada dibujo omitiendo el fonema inicial.

3.3.2 Omisión de sílabas en las palabras: en esta tarea se presenta una serie de dibujos, y el niño debe pronunciar el nombre de cada uno de ellos omitiendo la sílaba inicial o final, según se le indique.

\subsection{Descubrir si la sílaba inicial o final coincide con la de otra palabra}

En esta tarea se presentan oralmente pares de palabras de dos o tres sílabas, y el niño debe indicar si comienzan o terminan con la misma sílaba.

\subsection{Recomponer una palabra trisilábica}

En esta tarea se presentan oralmente palabras trisilábicas aisladas, manteniendo un breve intervalo entre cada una de las sílabas, el niño tiene que pronunciar la palabra correcta uniendo las sílabas que se presentaron secuencialmente.

En la fase postest (Tiempo 2), y en la fase de seguimiento (Tiempo 3), todos los sujetos fueron reevaluados con la Prueba de Segmentación Lingüística, PSL. A un año de finalizado el programa de estimulación (Tiempo 4) se les administró a todos los niños, una prueba de lectura de palabras (adaptación de la prueba utilizada por Serrano, 2005), para tener una medida del desempeño en lectura al finalizar el primer año de escuela. Esta prueba se compone de un total de 26 ítems (14 palabras bisilábicas y 12 trisilábicas) entre los que se encuentran palabras con estructura silábica simple, palabras con grupo consonántico y palabras con estructuras complejas, listadas en orden de dificultad creciente.

Todos los test fueron administrados de manera individual dentro del horario escolar, en un salón tranquilo del centro escolar.

Los sujetos fueron emparejados por la variable dependiente NCF $(p=.605)$, controlando, además, que no difirieran en vocabulario receptivo $(p=.809)$, inteligencia fluida $(p=.223)$, edad en meses $(p=.219)$ ni género $(p=.645)$. Posteriormente, cada par de sujetos fue asignado por sorteo a una de las 2 condiciones del estudio: grupo experimental (GE) y grupo control (GC). El GE estuvo compuesto por 4 niñas y 7 varones, y el GC por 4 niñas y 6 varones, lo que contribuyó a la validez interna del estudio (Schaughnessy, Zechmeister \& Zechmeister, 2007).

Los sujetos del grupo experimental $(n=11)$ participaron de un programa de música, centrado en favorecer el desarrollo de habilidades rítmicas, mediante ejercicios que requerían un manejo eficiente de la duración y la precisión. Las actividades se apoyaron en la percusión y acompañamiento rítmico de cantos africanos, juegos de ritmos con accesorios (claves, vasos plásticos, accesorios de percusión) y lectura musical de estructuras rítmicas simples, apoyados con notación gráfica. Se completaron 32 encuentros con una frecuencia de dos clases a la semana de 30 minutos cada una. Los niños del GC $(n=10)$ interpretaron canciones infantiles sin 
entrenamiento específico en habilidades rítmico-musicales durante el mismo periodo y con la misma frecuencia que los sujetos del GE.

\section{Características de las actividades musicales}

El programa de estimulación de la CF a través de la música se estructuró en dos módulos de 16 sesiones cada uno, y estuvo a cargo de una Licenciada en música y de dos estudiantes de psicología. Durante el primer módulo (16 sesiones), se implementaron actividades musicales de percusión, acompañamiento rítmico de canciones y juegos de ritmos con accesorios de percusión. Todas las actividades fueron realizadas de manera grupal. La complejidad de las tareas fue aumentando gradualmente $\mathrm{y}$, en algunas sesiones, la dificultad fue modulada en función del desempeño de los participantes.

El foco de las actividades musicales siempre estuvo puesto en la estructura rítmica del material musical que se utilizó, y en la percepción y manipulación de las propiedades temporales de los eventos sonoros, tales como la duración, la secuenciación y el ritmo. No obstante, en varias de éstos se utilizaron registros de audio para apoyar las actividades y los juegos musicales. Se buscó que el repertorio de canciones, rimas u otros juegos estuviesen mayormente en lenguas distintas al español, con el objetivo de que las actividades musicales no estuviesen interferidas por la estructura de la lengua materna de los niños, y los participantes se centraran, mayormente, en la estructura rítmica de los ejercicios. Así, el acompañamiento rítmico, los juegos de manos y juegos de ritmos con vasos y otros accesorios, se apoyaron principalmente en el recitado de rimas en portugués, o en canciones infantiles de origen africano, en dialecto lingala. Al mismo tiempo, se emplearon diversos juegos de iniciación musical para enseñar distintas nociones musicales (tempo, pulso, acento métrico, accelerando, diminuendo, ritardando y silencios). Cada uno de los juegos contemplaba distintas variantes de ejecución, y se realizaban en distintas velocidades y dinámicas de modo de aumentar las demandas y la complejidad de los ejercicios. En el segundo módulo (16 sesiones) se introdujeron actividades de reproducción de patrones rítmicos en 2 tiempos, siguiendo un modelo presentado por el experimentador, y ejercicios de lectura rítmica en 2, 3 y 4 tiempos, alternando silencios en el primer o último tiempo. En este módulo también se les enseñó a leer patrones rítmicos simples, pero debido a que adquirir conocimientos y destrezas relacionadas con la lectura musical tradicional implica un grado de dificultad considerable para la franja etaria con la que trabajamos, utilizamos notación musical gráfica. Este tipo de notación se basa en dibujos y símbolos, donde cada símbolo representa una duración, un ataque y un timbre determinado, y permite trabajar, casi de modo exclusivo, los aspectos rítmicos de una estructura.

Los niños del GC participaron de un taller en el que interpretaron canciones infantiles junto a los referentes adultos, acompañados con el audio de las canciones mediante un reproductor de $\mathrm{mp} 3$, durante el mismo periodo y con la misma frecuencia que los sujetos del GE. Los niños del GC no recibieron entrenamiento específico en música, y estas sesiones no contemplaban ninguna actividad de práctica musical.

Previo a analizar el efecto de la estimulación se controló que no existieran diferencias en la asistencia de los niños para cada una de las condiciones $(p=.918)$.

\section{Resultados}

Dado el tamaño muestral de nuestro estudio $(N=21)$, no se asumió ninguna forma particular de distribución, por lo que se buscaron diferencias entre los grupos a partir de pruebas no paramétricas, y se utilizaron los rangos promedio para el análisis comparativo entre los grupos (Bologna, 2012). Todas las comparaciones se realizaron con la prueba $U$ de Mann Whitney. Como medida de tendencia central se reportará la mediana, dado que no se ve afectada 
por la presencia de valores extremos, y se reportarán los percentiles 25 y 75 (Schaughnessy et al., 2007).

En el Tiempo 2 (post-entrenamiento), para comparar los grupos se trabajó con la variable cambio, calculada como la ganancia de puntaje entre el Tiempo 1 y Tiempo 2, y como medida de tamaño del efecto se calculó la $r$ de Rosenthal.

En la Tabla 1 se puede apreciar que las ganancias diferenciales en NCF fueron estadísticamente significativas entre los grupos en NCF $(r=0.69 ; p=.001)$, y en 4 de las 5 tareas evaluadas: $\operatorname{segmentar}(r=0.80 ; p=<.001)$; aislar $(r=0.47 ; p=.029)$; omitir $(r=.057 ; p=.008)$ y recomponer $(r=0.45 ; p=.043)$. Las diferencias en ganancia no fueron significativas en la tarea de descubrir diferencias entre las palabras $(p=.085)$

Tabla 1

Ganancias diferenciales en NCF y tamaño del efecto

\begin{tabular}{|c|c|c|c|c|c|c|c|c|}
\hline & $\begin{array}{c}\text { Grupo } \\
\text { Experimental }\end{array}$ & $\begin{array}{c}\text { Grupo } \\
\text { Control }\end{array}$ & & & & & & \\
\hline Tiempo 2 & Mediana & $\mathrm{P} 25-75$ & Mediana & P25-75 & $U$ & $Z$ & $p$ & $r$ \\
\hline $\mathrm{NCF}$ & 27 & 23-31 & .50 & $\begin{array}{c}-1.00- \\
7.00\end{array}$ & 9.500 & -3.206 & .001 & .69 \\
\hline Segmentar & 6 & $5-7$ & .00 & $\begin{array}{c}-3.00- \\
3.00\end{array}$ & 3.000 & -3.681 & .000 & .80 \\
\hline Aislar & 2 & $.00-4$ & .00 & $\begin{array}{l}-1.00- \\
1.00\end{array}$ & 24.500 & -2.189 & .029 & .47 \\
\hline Omitir & 12 & $10-18$ & 1.50 & $\begin{array}{c}-1.00- \\
7.00\end{array}$ & 18.000 & -2.616 & .008 & .57 \\
\hline Descubrir & 4 & $2-4$ & 2.00 & $\begin{array}{c}-3.00- \\
2.00\end{array}$ & 30.500 & -1.758 & .085 & $\ldots$ \\
\hline Recomponer & 1 & $.00-2$ & -1.00 & $\begin{array}{c}-1.00- \\
0.0\end{array}$ & 2.500 & -2.064 & .043 & .45 \\
\hline
\end{tabular}

NCF (75puntos): Segmentar (18 puntos); Aislar (6 puntos); Omitir (26 puntos); Descubrir (20 puntos);

Recomponer (5 puntos)

En el Tiempo 3, a los efectos de conocer si las diferencias en NCF entre los grupos se habían mantenido en el tiempo, los niños fueron reevaluados con el test PSL a su ingreso a primer año de escuela.

En la Tabla 2 se observa que, habiendo transcurrido cinco meses de finalizada la estimulación, se mantuvieron las diferencias estadísticamente significativas entre los grupos en NCF $(p=.003)$, y en las puntuaciones totales de las tareas de segmentación $(p=.002)$ y omisión $(p=.003)$. Las diferencias significativas en las puntuaciones totales de las tareas descubrir semejanzas entre las palabras $(p=.085)$; aislar sonidos $(p=.973)$, y recomponer palabras trisilábicas ( $p=.173)$, no se mantuvieron en el tiempo. 
Tabla 2

Desempeño en NCF y por tipo de tarea fonológica

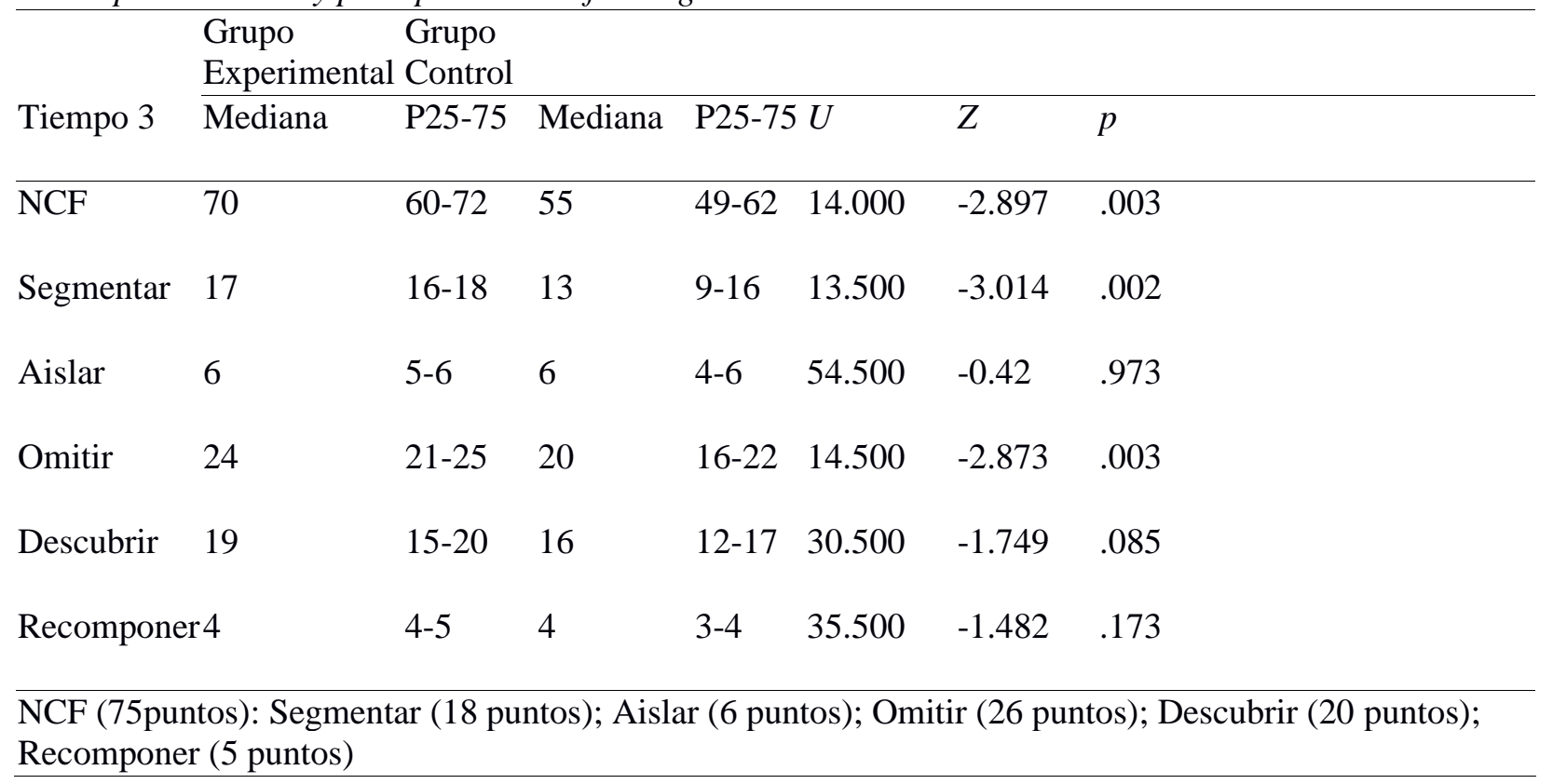

En el Tiempo 4, un año después de finalizado el entrenamiento, se administró una prueba de lectura de palabras (adaptación de la prueba utilizada por Serrano, 2005) y se calculó una medida de eficiencia lectora, analizando la cantidad de palabras leídas correctamente por minuto. En esta fase se obtuvieron datos de 19 participantes (10 del grupo experimental y 9 del grupo control). En la Tabla 3 se observa que la diferencia en eficiencia lectora fue estadísticamente significativa entre los grupos $(p=.035)$.

Tabla 3

Palabras leídas correctamente por minuto

\begin{tabular}{|c|c|c|c|c|c|c|c|}
\hline & $\begin{array}{l}\text { Grupo } \\
\text { Experimental }\end{array}$ & $\begin{array}{l}\text { Grupo } \\
\text { Control }\end{array}$ & & & & & \\
\hline $\begin{array}{l}\text { Tiempo } \\
4\end{array}$ & Mediana & P25-75 & Mediana & P25-75 & $U$ & $Z$ & $p$ \\
\hline $\begin{array}{l}\text { Cantidad } \\
\text { de } \\
\text { palabras }\end{array}$ & 123 & $17-25$ & 11 & $8-13$ & 19.500 & -2.095 & .35 \\
\hline
\end{tabular}

Total: 26 palabras

\section{Discusión}

El objetivo principal de este estudio fue analizar los efectos de un Programa de estimulación de la CF a través de una intervención musical centrada en el desarrollo de habilidades rítmicas, en un grupo de niños uruguayos de 5 años, de lo que no contamos con evidencia para el español del Uruguay.

Las evaluaciones se llevaron a cabo en cuatro momentos diferentes; Tiempo 1 iniciado el primer semestre escolar del último año de educación inicial; Tiempo 2 al finalizar el último año de educación inicial; Tiempo 3, al ingreso de los niños a primer año de escuela, y Tiempo 4, al finalizar el primer año de escuela.

Los resultados obtenidos del análisis de los datos en el Tiempo 2 mostraron que los niños 
que participaron durante cuatro meses del Programa de estimulación musical tuvieron un incremento superior al GC en NCF. Al mismo tiempo, los resultados por tipo de tarea fonológica también mostraron que el GE obtuvo una ganancia mayor al GC en 4 de las 5 tareas evaluadas (segmentar, aislar, omitir y recomponer). No se observaron cambios significativos entre los grupos en la tarea de descubrir semejanzas entre las palabras.

Una tercera evaluación se llevó a cabo al ingreso de los niños al primer año de escuela. Las diferencias estadísticamente significativas entre los grupos se mantuvieron en el NCF, y en 2 de las 5 tareas fonológicas evaluadas; segmentar y omitir. Esto pone en evidencia que los efectos del programa resultaron duraderos y no fueron compensados por el desarrollo espontáneo de los niños del GC.

El aumento de puntuaciones observado por el GC en esta fase en las tareas de aislar sonido inicial y final en una palabra y recomponer palabras trisilábicas presentadas secuencialmente en sílabas se corresponde con lo señalado por Jiménez y Ortiz (2008) sobre el incremento que debería observarse en las puntuaciones obtenidas en esta prueba, PSL, en el transcurso de, al menos, 7 meses entre una aplicación y la siguiente, debido a la evolución importante que debería experimentar el desarrollo de la $\mathrm{CF}$ durante ese periodo de tiempo. Al mismo tiempo, si se consideran las puntuaciones totales en las tareas de aislar y recomponer, las diferencias podrían explicarse por el puntaje ideal de cada una. La puntuación máxima para la tarea de aislar es de 6 puntos y la puntuación máxima para la tarea de recomponer es de 5 puntos, lo que sugiere que avances pequeños del GC en estas tareas, llevaron a que las diferencias significativas entre los grupos observadas en el Tiempo 2 desaparecieran en el Tiempo 3.

En cuanto al desempeño obtenido por ambos grupos en la tarea Descubrir semejanzas entre las palabras, tomando en cuenta las puntuaciones totales, existen dos posibles explicaciones: En función de la adquisición temprana de las habilidades fonológicas, algunos autores consideran que se trata de una tarea sencilla, en la que el niño requiere apenas un grado de consciencia sobre la estructura fonológica del lenguaje oral, y que el desempeño en este tipo de tareas no revela diferencias sustanciales entre grupo etarios (Defior \& Serrano, 2014). Por otra parte, la ausencia de diferencias en esta tarea también podría ser atribuida a las características del programa de estimulación. En éste las actividades musicales no estuvieron centradas en tareas de identificación de semejanzas o diferencias entre estructuras melódicas, y además se procuró no explotar las regularidades del español, controlando expresamente este componente al valerse de juegos rítmicos en otros dialectos o lenguas, para que los niños no se centraran en la estructura fonológica de su lengua oral durante el entrenamiento. Mientras, los niños del GC estuvieron siendo estimulados en esta habilidad, a través de las canciones infantiles. Este resultado es similar al reportado en el estudio de Herrera et al (2007), en el que sólo el grupo de niños que participó de un programa de entrenamiento de la CF que incluía la utilización de canciones infantiles, experimentó un incremento en el desempeño en tareas de identificar la rima entre dos palabras.

Tomados en su conjunto, los resultados de este estudio se corresponden en general con los de otras investigaciones realizadas con niños preescolares hispanoparlantes, que avalan la existencia de un determinado desarrollo evolutivo en las tareas de CF, en orden creciente de dificultad y diferentes momentos de emergencia durante el desarrollo (contar, aislar, adicionar, omitir y sintetizar) (de la Calle et al., 2016; Defior \& Serrano, 2014; Jiménez \& Ortiz, 2008; Nohales \& Giménez, 2014; Villagrán et al., 2011).

Por otra parte, los resultados de este estudio muestran una correspondencia con investigaciones previas en las que se ha reportado que la percepción y producción del ritmo está estrechamente vinculado con la habilidad de segmentación fonológica y la detección de sílabas, y que la capacidad para percibir y manipular intervalos de tiempo en una secuencia de sonidos es fundamental para un desempeño eficiente en tareas fonológicas (Dellatolas, Watier, Le Normand, Lubart, \& Chevrie-Muller, 2009; François et al., 2012; Moritz et al, 2013). Al mismo tiempo, se 
observa que el buen desempeño alcanzado por el GE en las tareas de omitir sonidos vocálicos y consonánticos y sílabas al inicio y al final de una palabra encuentra una conexión directa con las actividades de reproducción y lectura de patrones rítmicos con introducción de silencios en el primer o último tiempo, trabajadas durante el segundo módulo del entrenamiento musical específico. Por lo tanto, se puede sugerir que el entrenamiento en este tipo de actividades musicales resultó ser efectivo para una de las tareas más complejas para niños de esta franja etaria, y que requieren de algún grado de instrucción.

Con el objetivo de analizar el desempeño en precisión y velocidad lectora, un año después de finalizado el entrenamiento, se tomó una medida de lectura de palabras. En esta fase se obtuvieron datos de 19 participantes (10 del grupo experimental y 9 del grupo control). Los análisis mostraron que la tasa de palabras leídas correctamente por minuto por los niños del GE fue superior a los sujetos del GC. Estos resultados son consistentes con la amplia literatura que sostiene que la CF es un precursor clave para la adquisición de la lectura, en lenguas que cuentan con un sistema alfabético (Alegría, 2006; Bravo et al., 2011; Caravolas et al., 2012; Castles et al., 2018; Defior, 2014; Dehaene, 2017; Melby-Lervag et al., 2012; Morais, 2016)

Ahora bien, es necesario y relevante profundizar en la influencia que pudo haber tenido el entrenamiento musical en la atención y concentración, así como en el funcionamiento ejecutivo de los niños (Degé, Kubicek, \& Schwarzer, 2011; Frischen, Schwarzer, \& Degé, 2019; Kraus \& Chandrasekaran 2010; Moreno et al., 2011). Dada las características de las actividades del programa, y las demandas del tipo de actividades musicales empleadas, es necesario indagar en los efectos del entrenamiento en la memoria de trabajo, control inhibitorio, flexibilidad cognitiva, concentración y atención de los niños, lo que pudo haber facilitado la resolución de las tareas fonológicas de mayor demanda y complejidad (Thomas, 2016). Por lo tanto, la mediación de las funciones ejecutivas entre el entrenamiento musical y las habilidades lectoras debería ser considerada en investigaciones futuras.

Los resultados de esta investigación van en la misma dirección que la literatura actual que sostiene que la $\mathrm{CF}$ puede ser estimulada en niños prelectores a través de la música, y se suma a la evidencia de que el ritmo es un componente importante a la hora de diseñar actividades musicales para implementar en los niveles iniciales (Bolduc \& Rondeau, 2015; Gordon et al., 2015; Hansen et al., 2014; Moritz et al., 2013; Slater et al., 2014).

A la fecha, este es el primer estudio que aborda las relaciones entre habilidades rítmicas y desarrollo de la CF en niños uruguayos de 5 años con desarrollo típico, hablantes de español, y muestra que el entrenamiento musical que explota los parámetros de duración vinculados con el ritmo musical tiene efectos beneficiosos y duraderos tanto sobre el desarrollo de la $\mathrm{CF}$, como sobre la lectura fluida de palabras un año después de iniciada la instrucción formal.

\section{Limitaciones del estudio y líneas futuras a seguir}

A pesar de su carácter novedoso, este estudio presenta algunas limitaciones que deben ser atendidas en la investigación futura. Una de ellas es el tamaño de la muestra utilizado, que no permite realizar inferencias a nivel poblacional. Esto implica que los resultados deben analizarse teniendo en cuenta su valor esencialmente exploratorio. Se requerirá que la investigación futura incluya un mayor número de sujetos y grupos, así como también diversifique las características de la población a estudiar. Por otra parte, si bien el diseño utilizado en este estudio, y los análisis realizados nos permiten atribuir, con algún grado de certeza, el efecto del entrenamiento en las habilidades de $\mathrm{CF}$, hubiese sido interesante contar con un instrumento que establezca un nivel de base en habilidades musicales, tanto melódicas como rítmicas, al inicio de la investigación. Al mismo tiempo, tal como se observó en el apartado de la discusión, hubiese sido relevante contemplar una medida de funcionamiento ejecutivo que nos permitiera analizar si estas habilidades fueron estimuladas directamente a través del entrenamiento musical, y que pudiesen 
dar cuenta de los resultados obtenidos en CF.

Se espera que futuras investigaciones puedan atender estas limitaciones, ampliando y diversificando la muestra; contando con una medida de funcionamiento ejecutivo y de habilidades musicales específicas para la franja etaria utilizada en este estudio. Al mismo tiempo se espera que nuevos estudios continúen profundizando en esta línea de investigación sobre la relación entre las habilidades musicales y las habilidades lectoras iniciales en niños que aprenden a leer en español, y que aporten al diseño de programas de educación musical para que sean implementados durante los años de educación inicial

\section{Referencias}

Alegría, J. (2006). Por un enfoque psicolingüístico del aprendizaje de la lectura y sus dificultades-20 años después. Infancia y aprendizaje, 29(1), 93-111.

Anvari, S. H., Trainor, L. J., Woodside, J., \& Levy, B. A. (2002). Relations among musical skills, phonological processing, and early reading ability in preschool children. Journal of Experimental Child Psychology, 83(2), 111-130. doi: 10.1016/S0022- 0965(02)00124-8

Bolduc, J. (2009). Effects of a music programme on kindergartners' phonological awareness skills. International Journal of Music Education, 27(1), 37-47. doi: $10.1177 / 0255761408099063$

Bolduc, J., \& Montésinos-Gelet, I. (2005). Pitch Processing and Phonological Awareness. Psychomusicology: A Journal of Research in Music Cognition, 19(1), 3-14. doi: $10.1037 / \mathrm{h} 0094043$

Bolduc, J. \& Rondeau, J. (2015). Rythmons les apprentissages! Revue Langage et pratiques, 56, $15-22$.

Bologna, E. (2012). Estadística para psicología y educación. Córdoba, Argentina: Editorial Brujas.

Bravo, L., Villalón, M., \& Orellana, E. (2011). La conciencia fonológica y la lectura inicial en niños que ingresan a primer año básico. Psykhé, 11(1), 175-182.

Caravolas, M., Lervåg, A., Mousikou, P., Efrim, C., Litavský, M., Onochie-Quintanilla, E., ... \& Seidlová-Málková, G. (2012). Common patterns of prediction of literacy development in different alphabetic orthographies. Psychological science, 23(6), 678-686. doi: $10.1177 / 0956797611434536$

Castles, A., Rastle, K., \& Nation, K. (2018). Ending the Reading Wars: Reading Acquisition From Novice to Expert. Psychological Science in the Public Interest, 19(1), 5-51. doi: $10.1177 / 1529100618772271$

Cuadro, A. (2010). La lectura y sus dificultades: la dislexia evolutiva. Montevideo: Grupo Magro.

Cuadro, A., \& Trías, D. (2008). Desarrollo de la conciencia fonémica: Evaluación de un programa de intervención. Revista Argentina de Neuropsicología, 11(8), 1-8.

Defior, S. (2014). Procesos implicados en el reconocimiento de las palabras escritas. Aula, 20, 25-44.

Defior, S., \& Serrano, F. (2011). La conciencia fonémica, aliada de la adquisición del lenguaje escrito. Revista de logopedia, foniatría y audiología, 31(1), 2-13 doi: 10.1016/S02144603(11)70165-6

Defior, S., \& Serrano, F. (2014). Aspectos diacrónicos y sincrónicos del español: Relación con la adquisición del lenguaje escrito. Estudios de Psicología, 35(3), 450-475.

Degé, F., \& Schwarzer, G. (2011). The effect of a music program on phonological awareness in preschoolers. Frontiers in psychology, 2(124). doi: 10.3389/fpsyg.2011.00124.

Degé, F., Kubicek, C., \& Schwarzer, G. (2011). Music lessons and intelligence: A relation mediated by executive functions. Music Perception: An Interdisciplinary Journal, 29(2), 
195-201. doi: 10.1525/mp.2011.29.2.195

Dehaene, S. (2017). El cerebro lector. Buenos Aires: Siglo XXI.

de la Calle Cabrera, A. M., Villagrán, M. A., \& Guzmán, J. I. N. (2016). Desarrollo evolutivo de la conciencia fonológica: ¿Cómo se relaciona con la competencia lectora posterior? Revista de investigación en logopedia, 6(1), 22-41.

Dellatolas, G., Watier, L., Le Normand, M. T., Lubart, T., \& Chevrie-Muller, C. (2009). Rhythm reproduction in kindergarten, reading performance at second grade, and developmental dyslexia theories. Archives of Clinical Neuropsychology, 24(6), 555-563.

Domínguez, P., Nasini, S., \& Teberosky, A. (2013). Juegos de lenguaje y aprendizaje del lenguaje escrito. Infancia y aprendizaje, 36(4), 501-515. doi: 10.1174/021037013808200276

Dunn, Ll. M., Dunn, L. M. \& Arribas, D. (2006). PPVT-III PEABODY: Test de vocabulario en imágenes: manual. Madrid: TEA Ediciones.

Flaugnacco, E., Lopez, L., Terribili, C., Zoia, S., Buda, S., Tilli, S., ... \& Schön, D. (2014). Rhythm perception and production predict reading abilities in developmental dyslexia. Frontiers in human neuroscience, 8, 392. doi: 10.3389/fnhum.2014.00392

François, C., Chobert, J., Besson, M., \& Schön, D. (2012). Music training for the development of speech segmentation. Cerebral Cortex, 23(9), 2038-2043. doi: 10.1093/cercor/bhs180

Frischen, U., Schwarzer, G., \& Degé, F. (2019). Comparing the effects of rhythm-based music training and pitch-based music training on executive functions in preschoolers. Frontiers in Integrative Neuroscience, 13, 41. doi: 10.3389/fnint.2019.00041

Gordon, R. L., Fehd, H. M., \& Mc Candliss, B. D. (2015). Does music training enhance literacy skills? A meta-analysis. Frontiers in psychology, 6, 1777. doi: 10.3389/fpsyg.2015.01777

Goswami, U. (2012)."Language, music, and children's brains: a rhythmic timing perspective on language and music as cognitive systems," En P. Rebuschat, M. Rohrmeier, J. Hawkins, and I. Cross. (Eds.), Language and Music as Cognitive Systems (pp. 292-301). Oxford, UK: Oxford University Press.

Goswami, U. (2012b). Neuroscience and education: Can we go from basic research to translation? A possible framework from dyslexia research. En: BJEP Monograph Series II, Number 8-Educational Neuroscience (Vol. 129, No. 142, pp. 129-142). British Psychological Society.

Goswami, U., Wang, H.L., Cruz, A., Fosker, T., Mead, N. \& Huss, M. (2010). Languageuniversal sensory deficits in developmental dyslexia: English, Spanish, and Chinese. Journal of Cognitive Neuroscience, 23(2), 325-337. doi: 10.1111/j.14677687.2010.00955.x

Gromko, J. E. (2005). The effect of music instruction on phonemic awareness in beginning readers. Journal of Research in Music Education, 53(3), 199-209. doi: 10.1177/002242940505300302

Hansen, D., Bernstorf, E., \& Stuber, G. M. (2014). The music and literacy connection. London, UK: Rowman and Littlefield.

Herrera, L., Defior, S., \& Lorenzo, O. (2007). Intervención educativa en conciencia fonológica en niños prelectores de lengua materna española y tamazight. Comparación de dos programas de entrenamiento. Infancia $y$ aprendizaje, 30(1), 39-54. doi: 10.1174/021037007779849718

Herrera, L., Hernández-Candelas, M., Lorenzo, O., \& Ropp, C. (2014). Influencia del entrenamiento musical en el desarrollo cognitivo y lingüístico de niños de 3 a 4 años. Revista de psicodidáctica, 19(2), 367-386.

Holliman, A. J., Wood, C., \& Sheehy, K. (2010). The contribution of sensitivity to speech rhythm and non-speech rhythm to early reading development. Educational Psychology, 30(3), 247-267. doi: 10.1080/01443410903560922 
Jiménez, J. E. \& Ortiz, M. R. (2008). Conciencia fonológica y aprendizaje de la lectura: Teoría, evaluación e intervención. Madrid: Síntesis.

Kjeldsen, A. C., Karna, A., Niemi, P., Olofsson, A., \& Witting, K. (2014). Gains from training in phonological awareness in kindergarten predict reading comprehension in grade 9. Scientific Studies of Reading, 18(6), 452-467.

Kraus N, \& Chandrasekaran, B. (2010). Music training for the development of auditory skills. Nature Reviews Neuroscience, 11, 599-605.

Lamb, S. J., \& Gregory, A. H. (1993). The relationship between music and reading in beginning readers. Educational Psychology, 13(1), 19-27. doi: 10.1080/0144341930130103

Lucas, J., \& Gromko, J. (2007). The Relationship of Musical Pattern Discrimination Skill and Phonemic Awareness in Beginning Readers. Contributions to Music Education, 34, 9-17

Lundetræ, K., \& Thomson, J. M. (2018). Rhythm production at school entry as a predictor of poor reading and spelling at the end of first grade. Reading and writing, 31(1), 215-237.

Melby-Lervåg, M., Lyster, S.-A. H., \& Hulme, C. (2012). Phonological skills and their role in learning to read: A meta-analytic review. Psychological Bulletin, 138(2), 322-352. doi: $10.1037 / \mathrm{a} 0026744$

Morais, J. (2016). Lire, écrire et être libre: de l'alphabétisation à la démocratie. Paris: Odile Jacob.

Moreno, S., Bialystok, E., Barac, R., Schellenberg, E. G., Cepeda, N. J., \& Chau, T. (2011). Short-term music training enhances verbal intelligence and executive function. Psychological science, 22(11), 1425-1433. doi: 10.1177/0956797611416999

Moritz, C., Yampolsky, S., Papadelis, G., Thomson, J., \& Wolf, M. (2013). Links between early rhythm skills, musical training, and phonological awareness. Reading and Writing, 26(5), 739-769.

Nohales, P. S., \& Giménez, T. M. (2014). Secuencia evolutiva del conocimiento fonológico en niños prelectores. Revista de Logopedia, Foniatría y Audiología, 34(3), 118-128.

Patel, A. D. (2010). Music, language, and the brain. Oxford: Oxford University Press.

Peretz, I. (2018). Apprendre la musique: nouvelles des neurosciences. Paris: Odile Jacob.

Peynircioğlu, Z. F., Durgunoglu, A. Y., \& Úney Küsefog`lu, B. (2002). Phonological awareness and musical aptitude. Journal of Research in reading, 25(1), 68-80. doi: 10.1111/14679817.00159

Raven, J. C., Court, J. H., \& Raven, J. (1993). Test de matrices progresivas. Escalas coloreada, general y avanzada. Manual. Buenos Aires: Paidós.

Serrano, F. (2005). Disléxicos en español. Papel de la fonología y la ortografía. Tesis doctoral. Universidad de Granada.

Slater, J., Strait, DL., Skoe, E., O'Connell, S., Thompsonm E., et al. (2014). Longitudinal Effects of Group Music Instruction on Literacy Skills in Low-Income Children. PLOS ONE, 9(11): e113383. doi: 10.1371/journal.pone.0113383

Shgaugnessy, J., Zechmeister, E. \& Zechmeister, J. (2007). Métodos de investigación en psicología (7 $7^{\mathrm{a}}$ ed.). México DF: McGraw-Hill.

Tierney, A., White-Schwoch, T., MacLean, J., \& Kraus, N. (2017). Individual differences in rhythm skills: Links with neural consistency and linguistic ability. Journal of Cognitive Neuroscience, 29(5), 855-868.

Tierney, A., \& Kraus, N. (2013). Music training for the development of reading skills. Progress in brain research, 207, 209-241. doi: 10.1016/B978-0-444-63327-9.00008-4

Thomas, S. A. (2016). The Effect of Executive Function on emergent literacy skills a metaanalysis (Doctoral dissertation), University of Arizona.

Thomson, J., Huss, M., \& Goswami, U. (2006). Auditory perceptual training and developmental dyslexia: does it help? Poster presented at the 25th Rodin Remediation Conference, 1113 October, Georgetown University, Washington, DC. 
Torres, J. A. (2014). Conciencia fonológica en dos grupos de escolares que cursan primero básico de colegios municipales y particulares subvencionados (Doctoral dissertation), Pontificia Universidad Católica de Chile.

Villagrán, M. A., Consejero, E. M., Guzmán, J. I. N., Jiménez, I. M., \& Cuevas, C. A. (2011). Niveles de dificultad de la conciencia fonológica y aprendizaje lector. Revista de logopedia, foniatría y audiología, 31(2), 96-105. doi: 10.1016/S0214-4603(11)70177-2

Wood, C., Wade-Woolley, L., \& Holliman. A. J. (2009). Phonological awareness: Beyond phonemes. En C. Wood \& V. Connelly (Eds.), Contemporary Perspectives on Reading and Spelling. (pp. 7-23). London: Routledge.

Financiamiento: La investigación que da origen a los resultados presentados en la presente publicación recibió fondos de la Agencia Nacional de Investigación e Innovación, ANII, bajo el código POS_NAC_2015_1_109771

Contribución de los autores: a) Concepción y diseño del trabajo; b) Adquisición de datos; c) Análisis e interpretación de datos; d) Redacción del manuscrito; e) revisión crítica del manuscrito.

J.R.I ha contribuido en a,b,c,d,e ; K.M.T. en a,c,d,e.

Editora científica responsable: Dra. Cecilia Cracco 